\title{
AMENDMENTS
}

\section{Author Correction: Identification of Exo1-Msh2 interaction motifs in DNA mismatch repair and new Msh2-binding partners}

Eva M. Goellner, Christopher D. Putnam, William J. Graham V, Christine M. Rahal, Bin-Zhong Li and

Richard D. Kolodner (iD

Correction to: Nature Structural \& Molecular Biology https://doi.org/10.1038/s41594-018-0092-y, published online 30 July 2018.

In the version of this article initially published, some of the information in Supplementary Table 3 was incorrect. The correct Supplementary Table 3 is now provided online. The error has been corrected in the HTML version of the article.

Published online: 4 November 2019

https://doi.org/10.1038/s41594-019-0337-4

๑ The Author(s), under exclusive licence to Springer Nature America, Inc. 2019

\section{Author Correction: G-tract RNA removes Polycomb repressive complex 2 from genes}

Manuel Beltran, Manuel Tavares, Neil Justin, Garima Khandelwal, John Ambrose, Benjamin M. Foster, Kaylee B. Worlock, Andrey Tvardovskiy, Simone Kunzelmann, Javier Herrero (D), Till Bartke (D), Steven J. Gamblin, Jon R. Wilson (D) and Richard G. Jenner (D)

Correction to: Nature Structural \& Molecular Biology https://doi.org/10.1038/s41594-019-0293-z, published online 23 September 2019.

In the version of this article initially published, Fig. 4 included some errors. In Fig. 4c, the color for the left bar in each set of three bars (green) was incorrect; the correct color is orange (H2AK119ub, as in key). In Fig. 4d, top row, the downward error bars for H3K27me3 in the top middle plot $(F g f 11$ B) were incorrect; the correct s.d. in the negative direction is smaller for each. In Fig. $4 \mathrm{~d}$, bottom row, the far left downward error bar for HA-dCas9 in the left plot $(F g f 11 \mathrm{~A})$ was incorrect; the correct s.d. in the negative direction is larger. The errors have been corrected in the HTML and PDF versions of the article. 